\title{
Next Generation Sequencing in Diagnosis of Reed's Syndrome, or Hereditary Leiomyomatosis and Renal Cell Cancer (HLRCC)
}

\author{
Ashley Savage Becker ${ }^{1}$, Mónica Lara Anaya ${ }^{2}$, Munaf Al Kadhimi ${ }^{3}$ and Julio A Peguero ${ }^{3 *}$ \\ ${ }^{1}$ The University of Texas Health Science Center at Houston, McGovern Medical School, USA \\ ${ }^{2}$ Universidad del Noreste, USA \\ ${ }^{3}$ Oncology Consultants, Research Department, International Cancer Center, USA \\ *Corresponding author: Julio A Peguero, Oncology Consultants, Research Department, International Cancer Center, USA
}

Submission: 非 July 07, 2018; Published: 眥 August 17, 2018

\begin{abstract}
Summary
A 40-year-old woman with a past medical history of myomatosis and dysmenorrhea, who presented to her primary care physician with various complaints such as shortness of breath and fatigue, was found to have a large left renal mass. In April, 2014, she underwent a total left nephrectomy with removal of 13 lymph nodes, and pathology confirmed papillary renal cell carcinoma. One year later, she had recurrence of her disease in the subligamentary space attached to T12, which was also surgically removed in November, 2015. Although she showed all three classic symptoms of hereditary leiomyomatosis and renal cell cancer (HLRCC), which include cutaneous and uterine leiomyomas and papillary renal cell cancer, she remained undiagnosed for 20 months after her initial renal cancer diagnosis. Genetic testing, including next generation sequencing, was performed on the tumor, and the results were consistent with HLRCC. After starting treatment with bevacizumab and erlotinib in December, 2015, she has remained free of disease.
\end{abstract}

\section{Background}

Hereditary leiomyomatosis and renal cell cancer (HLRCC), or Reed's syndrome, is a rare but potentially life-threatening familial disorder. While cutaneous and uterine leiomyomas are typically easy to detect, physicians should be aware of the risk of kidney cancer in patients who present with one or both of these symptoms. The associated renal cell carcinoma in families with HLRCC is usually very aggressive, with a high rate of metastasis and poor prognosis, and develops at an early age.

\section{Case Presentation}

A 40-year-old Latin American woman with a past medical history of myomatosis and dysmenorrhea was in her usual state of health until the beginning of 2014 when she was planning a trip to ascend the Iztaccihuatl volcano in Mexico City. She presented to her primary care physician with significant shortness of breath upon regular daily activities, and reported a $10 \mathrm{gr} / \mathrm{dl}$ of hemoglobin. She then pursued her trip to the volcano, and was able to ascend an impressive 5000 feet. She returned to her primary care physician with complaints of gastroesophageal reflux, deep malaise and fatigue. Upon physical exam she was found to have a palpable left mid-quadrant abdominal mass. An ultrasound of the abdomen showed a left renal mass, and this visualization was confirmed by subsequent MRI.

She underwent a total left nephrectomy with thirteen lymph nodes in April, 2014. The mass measured $17 \mathrm{~cm} \times 14.7 \mathrm{~cm}$. The pathological description was consistent with papillary renal cell carcinoma with infiltration to the renal capsule but no surrounding perinephric tissues. The disease was grade moderately differentiated, and there were no positive margins and no lymphatic or vascular invasion. The left renal vein, artery and ureter were negative for neoplastic involvement. The immunohistochemistry profile was consistent with positive $\mathrm{Ck} 7$ and Vimentin, and negative for CK20 and Synaptophysin.

One year later in the summer of 2015 she complained of pain in her back. This prompted a follow up PET/CT and MRI, which revealed a soft tissue lesion in the subligamentary space attached to T12. After surgical resection of the soft tissue density and sampling of the bone, pathological examination of the soft tissue and bone found that they were both positive for papillary carcinoma.

Due to these manifestations, peripheral sampling for fumarate hydratase (FH) activity and gene testing for a de novo germline mutation were performed, giving the results that the $c .738+2 \mathrm{~T}>\mathrm{C}$ 
splice site variant in the FH gene destroys the canonical donor site in intron 5. This was consistent with the diagnosis of hereditary leiomyomatosis and renal cancer (HLRCC). In addition to the germline mutation, next generation sequencing of the tumor revealed the germ line event.

\section{Treatment, outcome and follow up}

The patient underwent successful treatment with bevacizumab and erlototinib starting in December, 2015, and she has remained free of disease.

\section{Discussion}

HLRCC, also known as Reed's Syndrome, is a dominantly inherited form of kidney cancer that is associated with cutaneous and uterine leiomyomas [1]. It is caused by germline mutations in the FH gene, causing loss of function of fumarate hydratase, an enzyme that operates in the mitochondrial Krebs cycle to convert fumarate to malate [2]. While the mechanism of tumorigenesis caused by mutations in FH is not entirely known, FH most likely acts as a tumor suppressor [2], and there is evidence that shows fumarate accumulation due to diminished $\mathrm{FH}$ activity may lead to a state of pseudohypoxia, creating a predisposition to tumors [3].

An association between cutaneous and uterine leiomyomas was first documented in successive generations of two families in 1973 by Reed et al., establishing an autosomal dominant inheritance pattern of the disease. These leiomyomata of the skin and uterus, which are benign smooth muscle tumors, are both highly penetrant in HLRCC families (above 70\%), and usually appear at a young age (under 30) [4,5]. Cutaneous leiomyomas, which arise from the pilo-arrector muscles surrounding hair follicles, are typically located on the trunk or limbs, and are often painful and sensitive to touch and cold temperatures [6]. Almost all (98\%) women with cutaneous leiomyomas also have uterine fibroids, which can cause dysmenorrhea and menorrhagia, and many (44\%) of these women must undergo a total hysterectomy before reaching age 30 (89\% total) [5].

Kidney cancer may be found in up to one-third of families with the FH mutation, and it develops predominantly as a type II papillary renal cell cancer (or collecting duct cancer).These renal cell carcinomas are usually unilateral, solitary, and have unique histology, with inclusion-like, orangiophilic nucleoli with a perinuclear halo [7]. Although the penetrance of kidney cancer in HLRCC families is less than that of the associated leiomyomas, renal cell cancer usually has an early age of onset (with a median age of detection at 44 years, but youngest age documented at 11 , and multiple patients in teens) $[5,8]$ and is highly aggressive, with a higher rate of distant metastasis than that observed in other hereditary renal cancer syndromes, such as Birt-Hogg-Dubé syndrome, with a 5 year survival rate of only $20 \%$ or less [9].

\section{Learning Points}

A. Patients with HLRCC present with cutaneous and uterine leiomyomas and are at increased risk for developing early onset, aggressive renal cell carcinoma.

B. HLRCC is a dominantly inherited familial disorder caused by mutations in the FH gene.

C. This case shows the importance of physician awareness of HLRCC in regard to recognizing potential aggressive metastatic disease in the presence of benign leiomyomas.

Next generation sequencing in the tumor may reflect germline mutations in patients with HLRCC.

\section{References}

1. Launonen V, Vierimaa O, Kiuru M, Isola J, Roth S, et al. (2001) Inherited susceptibility to uterine leiomyomas and renal cell cancer. Proc Natl Acad Sci U S A 98(6): 3387-3392.

2. Tomlinson I, Alam N, Rowan A, Barclay E, Jaeger E, et al. (2002) Germline mutations in $\mathrm{FH}$ predispose to dominantly inherited uterine fibroids, skin leiomyomata and papillary renal cell cancer. Nat Genet 30(4): 406410.

3. Pollard P, Wortham N, Tomlinson I (2003) The TCA cycle and tumorigenesis: the examples of fumarate hydratase and succinate dehydrogenase. Ann Med 35(8): 632-639.

4. Wei M (2005) Novel mutations in FH and expansion of the spectrum of phenotypes expressed in families with hereditary leiomyomatosis and renal cell cancer. J Med Genet 43(1): 18-27.

5. Toro J, Nickerson M, Wei M, Warren M, Glenn G, et al. (2003) Mutations in the fumarate hydratase gene cause hereditary leiomyomatosis and renal cell cancer in families in North America. Am J Hum Genet 73(1): 95-106.

6. Alam N, Olpin S, Leigh I (2005) Fumarate hydratase mutations and predisposition to cutaneous leiomyomas, uterine leiomyomas and renal cancer. Br J Dermatol 153(1): 11-17.

7. Merino MJ, Torres Cabala CA, Zbar B, Chian Garcia CA, Linehan WM (2003) Hereditary leiomyomatosis and renal cell carcinoma syndrome (HLRCC): clinical, histopathological and molecular features of the first American families described. Mod Pathol 16: 739.

8. Menko F, Maher E, Schmidt L, Middelton L, Aittomäki K, et al. (2014) Hereditary leiomyomatosis and renal cell cancer (HLRCC): renal cancer risk, surveillance and treatment. Familial Cancer 13(4): 637-644.

9. Gardie B, Remenieras A, Kattygnarath D, Bombled J, Lefevre S, et al. (2011) Novel FH mutations in families with hereditary leiomyomatosis and renal cell cancer (HLRCC) and patients with isolated type 2 papillary renal cell carcinoma. Journal of Medical Genetics 48(4): 226-234. 
NACS Nom Approaches
in Cancer Study

For possible submissions Click Here
Submit Article

\section{Novel Approaches in Cancer Study}

\section{Benefits of Publishing with us}

- High-level peer review and editorial services

- Freely accessible online immediately upon publication

- Authors retain the copyright to their work

- Licensing it under a Creative Commons license

- Visibility through different online platforms 